\title{
ENGINEERING IN MEDICINE
}

\author{
Amy Hsiao, Andrew Smith \\ Memorial University of Newfoundland \\ amy.hsiao@mun.ca
}

\begin{abstract}
This work presents a description and assessment of the first introductory course in biomedical engineering, the result of a two-year exchange between clinicians and researchers in the Faculties of Medicine and Engineering and Applied Science. The course is an integration of research into teaching, as well as the first step in incorporating engineering into Medicine. Through lectures, clinical shadowing, tours, critical reflection and a final project, students interested in healthcare experienced a unique, multidisciplinary learning environment in a seminar-style setting. The work will present student assignments, student feedback and student application of the key concepts in the course. An assessment of the defined learning outcomes for the course is also addressed. In presenting this work, the authors would also like to promote knowledge sharing of how engineering and medicine are integrated at other universities in the area of Biomedical Engineering, specifically as a major, a sub-discipline, or a multidisciplinary undergraduate degree.
\end{abstract}

Keywords: multidisciplinary teaching and learning, medical innovation, electrical engineering, mechanical engineering.

\section{INTRODUCTION}

The field of Biomedical Engineering is quite broad, but as a starting point, the first offering of this course at Memorial University (MUN) focused on current interests and capabilities represented by the Faculty of Medicine (FM) and Faculty of Engineering and Applied Science (FEAS). As such, the course included topics in medical informatics, devices, and diagnostics, engineering applications in orthopaedic and rehabilitation, simulation and education technologies for healthcare, and engineering innovation in medicine. A thematic scenario was also presented at the beginning of class, introducing a hypothetical patient "Johnny", a 30 year old male involved in a serious motor vehicle accident requiring acute medical care. Serious injuries result in activation of the pre-hospital system initiating Johnny's journey through the emergency room, radiology suite, operating room and hospital ward culminating in his eventual discharge. Each weekly lecture related back to the theme of "Johnny" and the final presentation and report prepared by the students also tied into this scenario.

The course was anchored by two faculty members, one associate professor from Mechanical Engineering, and one assistant professor from Medicine, an emergency medicine physician with a cross appointment in Electrical Engineering. In addition, at least one guest speaker from the engineering and medical community presented at each weekly 3-hour session, for a total of at least fifteen associated colleagues of the co-instructors sharing their expertise with the class of 23 students.

\section{COURSE DETAILS}

\subsection{Student Demographics}

The class of 23 students consisted of mostly (22) senior Term 8 students, selecting the course as an elective. The Term 8 students were mainly from the Electrical and Computer Engineering department and the Mechanical Engineering department, with one student in Ocean and Naval Architectural Engineering. At least one student applied to graduate school in Bioengineering or Biomedical Engineering, and at least one student in the course applied to Medical School.

\subsection{Shadowing and Tours}

All students were given the opportunity to participate in clinical shadowing in the emergency room at the Health Science Centre (General Hospital) or St. Claire's Hospital in St. John's. The ER physician would take 1-2 students per shift on designated dates, and students would sign up for their preferred dates. Students also had the opportunity as a class to don scrubs and tour the operating room for orthopaedic surgery. These opportunities were a result of the co-instructors' connections with the ER and Orthopedic Surgery departments. Students were required to sign Oaths of Confidentiality forms by Eastern Health in order to participate in these activities. 


\subsection{Participation}

Participation was a major component of the final assessment, worth $34 \%$ of the final mark, and consisted of individual contribution to the lectures and weekly events. One source of the participation mark included attendance and classroom discussion, worth $4 \%$. The second indicator of participation was based on weekly assignments focused on student reflection or application of course material. The course was divided into three modules, and each module had a participation component, as such:

- 15\% for Weeks 1-5: 5\% each of 3 assignments

- $10 \%$ for Weeks 6-11: $5 \%$ for each of 2 assignments

- 5\% for Week 12: $5 \%$ for each of 1 assignment

In addition, participation was defined in the course syllabus with a few examples to encourage class discussion and contribution:

Make observations that integrate concepts

- Share examples and current news items relevant to the topics covered in the course

- Ask questions that lead to revealing discussions

- Ask questions when concepts are unclear

- Be an active participant in lectures, on tours, and during demonstrations

- Pull your own weight on team projects and inclass activities

\subsection{Grading}

In addition to Participation, the other major components of the final assessment were Quizzes, worth $36 \%$ of the final mark, and Final Project, worth $30 \%$ of the final mark. There were three quizzes, each worth $12 \%$ in the course, with two offered during the term and one taking place during the Final Examination period. The Final Project was completed in self-selected teams and included a presentation component $(15 \%)$ using peer and instructor evaluation and a report component (15\%) using instructor evaluation only.

\subsection{Course Outline}

Figure 1 shows the schedule of exams, due dates, and lectures for the course.

\begin{tabular}{|c|c|}
\hline Dates & \\
\hline $\operatorname{Jan} 5-9$ & \\
\hline - Int & duction (1 week) - (Smith, Hsiao, Morgan) \\
\hline . & Introduction (Hsiao) \\
\hline 。 & Canadian health care system and Memorial Health Sciences profile (Smith) \\
\hline 。 & Patient Data \& Informatics, Transport Technologies, Hospital Operations (Smith, Morgan) \\
\hline
\end{tabular}

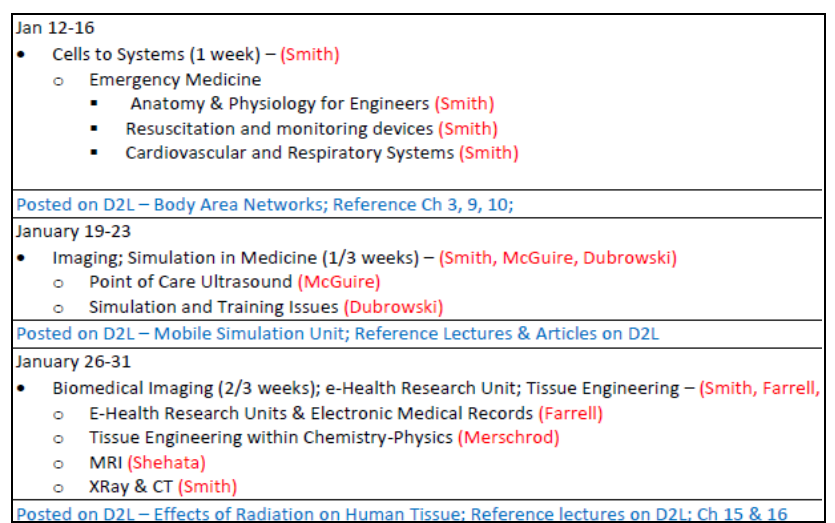

\begin{tabular}{|c|c|}
\hline \multirow{2}{*}{\multicolumn{2}{|c|}{$\begin{array}{l}\text { February 2-6 } \\
\text { - Biomedical Imaging ( }(3 / 3 \text { weeks) }- \text { (Smith, Maguire, Hollett, Shehata) } \\
\text { \& Nuclear Medicine \& Pet-CT (Maguire, Hollett) } \\
\text { - Medical Image Processing (Shehata) } \\
\text { - Biomechanics and Orthopedics (1/3 weeks) } \\
\quad \quad \text { Part I: Mechanics, Forces, and Modes of Motion (e.g. walking, running, swimming, cycling, gait) (Nakhla) } \\
\text { Read Chapters 2,3 }\end{array}$}} \\
\hline & \\
\hline & $\begin{array}{l}\text { 9-13 } \\
\text { QUIZ\#1 on Introduction and Biomedical Imaging (Smith) } \\
\text { Discussion of Final Project } \\
\text { Biomechanics and Orthopedics (1/3 weeks) } \\
\text { Part II: Mechanics, Forces, and Modes of Motion (e.g. walking, running, swimming, cycling, gait) (Nakhla) } \\
\text { Assignment \#4 }\end{array}$ \\
\hline \multicolumn{2}{|r|}{$\begin{array}{l}\text { Study for Quiz \#1 } \\
\text { February } 16-20 \text { WINTER BREAK }\end{array}$} \\
\hline & YY 16-20 WINTER BREAK \\
\hline
\end{tabular}

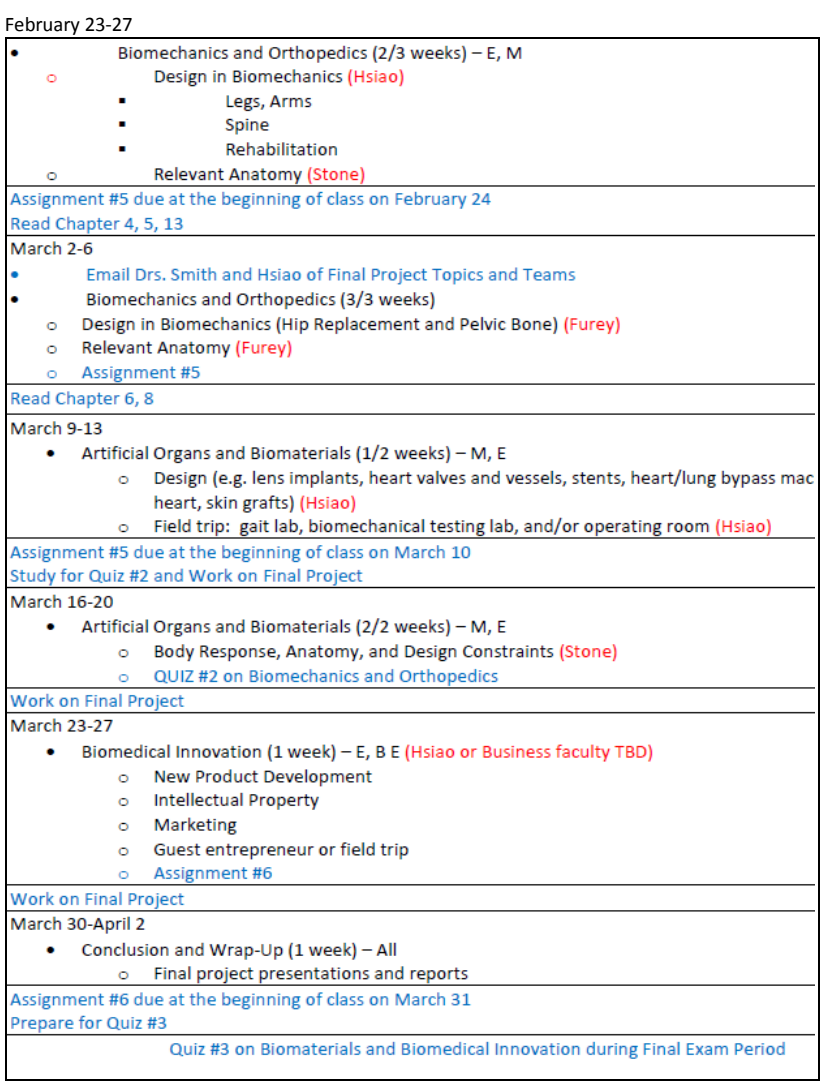

Fig. 1. Schedule of Lectures, Exams, and Due Dates

The progression of "Johnny" can be seen on a weekly basis. The co-instructors Hsiao and Smith also presented 
their own research in biomechanical testing and image processing, respectively, and invited medical students who had undergraduate engineering degrees to share their experiences on collaborative projects between Medicine and Engineering.

\section{ASSESSMENT}

There were three learning outcomes defined for students in this course, namely, that students would (1) be familiar with engineering applications in medicine, (2) be exposed to medical technology, innovation, and the organizational structure of healthcare, and (3), communicate technical information in a clear and effective manner. The three major components of assessment were defined as Participation, Quizzes, and Final Project, and the following sections present examples of each component.

\subsection{Example Assignments}

Assignments in the course were on a weekly or biweekly basis and focused on personal reflection. For example, the first assignment asked the students to prepare a journal review of an assigned research article. The following assignment asked students to reflect upon medical telecommunications:

Conceptualize the design of a mobile simulation unit incorporating telecommunications equipment using readily available vehicles, utility trailers, etc. Reflect on cost, portability, ability to support tele-simulation, functional space, etc.

Another assignment asked students to reflect upon biomechanics:

In class we discussed idealizing human anatomy (body segments, joints, and tendons) as machines in three respective categories, namely levers, wheels and axles, and pulleys. A number of anatomical examples were presented in class to illustrate the idealization concept. Provide two additional examples from human anatomy in one or more of the above categories. Support your answer with figures or sketches.

Oscar Pistorius is a South African sprint runner with double amputation. In 2008 he applied to compete in the able-bodied Summer Olympics in Beijing, China. His application was denied because he "runs differently". How does the gait cycle for an amputee differ from that of an ablebodied? Provide figure(s) and a brief discussion to highlight the main differences.

Another assignment asked students to reflect upon the major anatomical systems required to "do a leg press", as discussed by the orthopaedic surgeons in a guest lecture and tour.

Summarize the mechanism by which paralytic agents, ie succinylcholine, used in human anesthesia, paralyzes skeletal muscle. (1 page)

From your engineer's perspective, reflect upon the different aspects of the OR tour with Dr.

Furey where you saw engineering applied, where engineering could improve current capabilities, where engineering did not improve or facilitate how the OR functions. (1 page)

The final assignment asked students to identify social/demographic, economic, political, regulatory/legal, environmental or technological trends that could possibly present an opportunity for medical innovation.

\subsection{Quizzes}

The three quizzes in the course focused on each of the three modules: Biomedical Imaging, Biomechanics and Orthopaedics, and Biomaterials and Medical Innovation. Each quiz was 60-90 minutes and included multiple choice and essay questions. Using the second quiz as an example, students were asked to study by reflecting upon and preparing answers for these questions:

How do these major organ systems (i.e. nervous, muscular and skeletal systems) interact to produce movement?

- $\quad$ Kinetics

- Kinematics

- Motion capture and data synchronization: Gait

- "How to do a leg press"

List the four classes of materials used in medical devices, along with three advantages and disadvantages of each.

Describe which type of biomaterial you would select for the construction of the following implantable devices. Explain which properties will be important and why. More than one material can be used in the same device.

- Skin substitute

- $\quad$ Guidance tube for nerve regeneration

- Hip replacement stem

- Dental braces

- Urinary catheter

- Tissue-engineered bone

When selecting a biomaterial to be used as an orthopedic implant, what are some of the properties or characteristics of the material that should be considered?

Multiple choice questions were a bit more difficult as the material covered and the nature of the course could not really focus on detailed medical processes or terms. 


\subsection{Final Projects}

The topics for the final projects were selected by the students and included:

- Using Technology and Engineering Design for Improved Home Care for the Elderly

- Depression Monitoring Technology

- Business Feasibility Study: Consumer Exoskeletons

- Image Processing for Medicine

- Engineering Innovation in the Critical Care Process

- The Process of Diagnosing and Treating Prostate Cancer: Engineering in Medicine

- “Does Jane Live?" : Issues Surrounding a Car Accident

The rubrics for the peer evaluation were defined by four criteria for a total of 15 points, with students being able to select statements that they agreed with most completely (a qualitative Likert-like scale):

- Clarity of the Topic: The case or story presented was defined clearly and thoroughly.

1. I agree completely. The group presented a topic clearly with a story or case and went into good detail on it.

2. It was pretty clear and not a repeat of what we had already learned from our lectures.

3. The topic was not really developed and did not lend to any original ideas or discussion.

4. This was very poorful done. I was confused as to what they were presenting.

- Connection to the Course: The presentation explained revelant course ideas, subjects, concepts and the significance of Engineering in Medicine was clearly demonstrated.

1. I agree completely. The group used what we learned in the course and applied it to a specific interest point in great detail.

2. The group made good connections to the course topic.

3. The relevance was clear.

4. The relevance was implied but not clearly discussed.

5. I didn't see the significance of Engineering in Medicine or a personal reflection of the group's topic and how it relates to the course.

- Quality of the Presentation: The presentation was well-organized. The group used an appropriate number of slides, used their presentation time well, prepared what they were going to say, no spelling or proofreading issues. How was the overall delivery of the presentation?
1. Excellent presentation. Well-organized flow, good use of slides and other presentation tools.

2. A good presentation.

3. I felt the presentation could have used more preparation. Poorly prepared slides and the organization of thought was not there.

- Enthusiasm and Professionalism: The group members were engaging, prepared, and showed interest in their topic.

1. Absolutely. In the presentation and in the Q\&A, you could see the interest and enthusiasm in all the members.

2. This is true of some of the members, not all of them.

3. No, the interest and enthusiasm were not shown throughout the presentation.

\section{STUDENT FEEDBACK AND REFLECTION}

\subsection{Quality of Work}

Throughout the course, the students demonstrated quite a bit of enthusiasm and interest in the course material, as this was evident in the high level of interaction with speakers and positive class dynamics. Almost all of the students completed weekly assignments on a consistent basis (20 out of 23 ). The projects were the final, formal method of feedback and assessment of learning from the students. The topics they selected also demonstrated and applied their engineering discipline and interest to medicine.

\subsection{Online Survey Results}

At the end of the course, the co-authors created a survey to obtain anonymous student feedback on the first offering of the course for improvement, separate from the university's course evaluations. Although only four out of the 23 students responded to the voluntary survey, the results are presented here for discussion.

\section{Question 1: What attracted you to the course?}

- Applying Engineering knowledge to a nontraditional field such as industry or construction.

- I want to do biomedical engineering after I graduate and did several work term in biomed.

- Interested in a career in medicine.

- Multi - disciplinary applications of this course and how it would link both the engineering and medicine

Question 2: List three aspects you liked about the course. 
- Guess Speakers who gave their honest opinion on the topic at hand; The tools, and gadgets being brought to class really emphasized the material; Shadowing Dr. Smith and the trip to the OR.

- Loved the breadth of the material and how it exposed me to the huge variety of engineering in medicine. Loved the tours and field trips! They really enhanced the material as we were able to see how the topics were actually applied in real life. I liked that the class was a small group of mainly genuinely interested individuals as it stimulated conversations and resulted in more of a seminar style than lecture style classes

- Field visits; Exposure to medical technologies; Exposure to biological science

- This course was honestly the most interesting engineering course I have taken so far. I particularly enjoyed the diversity of the course and having different guest speakers almost every lecture. It combined a link to what engineering knowledge can develop into and how to apply this knowledge in the medical field. It was nice to learn about the innovations in medicine and in some assignments our opinions and ideas as students where valued in how to make those innovations better. Loved both ER and OR tours.

When asked what they did not like about the course, students listed the three-hour timeslot, overemphasis on specific areas like medical imaging and materials science, and inconsistent assignment rubrics.

Question 3: The course, "Engineering in Medicine":

- Generated, stimulated, or increased my interest in the subject area: $75 \%$ agree-strongly agree

- Emphasized the value of multidisciplinary collaboration: $100 \%$ agree-strongly agree

- Achieved balanced between engineering and medicine principles: $75 \%$ agree-strongly agree

- Clinical experiences enhanced the learning experience: $100 \%$ strongly agree

- Innovation and small business opportunities were integrated into the curriculum: 100\% agreestrongly agree

- Guest speakers were engaging and enhanced the learning: $100 \%$ agree-strongly agree

- Medicine theory was presented at the right level for my ability: $75 \%$ agree-strongly agree

- Engineering theory was presented at the right level for my ability: $100 \%$ agree-strongly agree

Question 4: The course instructors:

- Demonstrated mastery of the subject area: 4.75/5.0

- Communicated information effectively: 4.75/5.0

- Exhibited interest in my progress: $4.5 / 5.0$
- Demonstrated respect and a professional attitude during the session: $4.75 / 5.0$

- Conducted the training sessions and tours in an organized, efficient manner: 4.75/5.0

Question 4: How well do you think the learning outcomes were achieved?

- Be familiar with engineering applications in medicine: $100 \%$ agree-strongly agree

- Be exposed to medical technology, innovation, and the organizational structure of healthcare: $100 \%$ agree-strongly agree

- Communicate technical information in a clear and effective manner: $75 \%$ strongly agree

Question 5: I will be more likely to further my biomedical engineering knowledge via research or career opportunities in the future as a result of this course: $75 \%$ strongly agree

Overall the students were satisfied with the course, instructors, grading, and assignments.

\subsection{Challenges and Improvements}

One of the most important improvements for future offerings will be to have a set schedule of topics and committed speakers, such that the planning and delivery are not as heavily reliant on who the instructors are and their own professional connections. The student feedback alludes to some of these challenges when requesting more consistency in classtime, definition of rubrics, and depth of topic presentations.

Similarly, it may be important to consider this elective as two separate courses, one for Electrical and Computer Engineering students and one for Mechanical Engineering students, such that topics, assignments, and projects can have more depth and detail.

As the students were mainly a self-selected group who were interested in the topic, there was very little issue with low quality of work, missed assignments, or even the perception of the elective being "easy" or "non-technical", and it would be important to maintain this criteria in the future.

Finally, there is quite a bit of demand from students to have this type of course, so the challenge for future offerings is to meet that demand with the rigor, learning experience, and timing in their respective programs such that Questions 4 and 5 are achieved and realized in a very effective manner. This is reflected in one of the survey responses to Question 5:

"The one big thing that I do hope is that this course actually does continue for future years! It would be a 
shame if it doesn't. Thank you for such an interesting experience!"

\section{CONCUSION}

In the first offering of "Engineering in Medicine", the innovative, educational aspects of the course were the integrated, multidisciplinary presentations of the topic, the introduction of a theme that connected all of these topics from Electrical and Computer Engineering to Mechanical Engineering, and the active learning opportunities in and outside of the traditional classroom. The passion and enthusiasm that were demonstrated and experienced in this first offering should be balanced with more consistent personnel, topics, and assessment rubrics, tools, and plans in future offerings.

\section{Acknowledgements}

A Hsiao and A. Smith would like to acknowledge our colleagues who were enthusiastic, well-prepared and interactive guest speakers in the course and helped to bring depth and breadth to the first offering.

\section{References}

[1] John D. Enderle and Joseph D. Bronzino, Introduction to Biomedical Engineering Academic Press, 2011 (3rd ed.), 1272 pp. \{ISBN: 978-0123749796\} 